Itinéraires Itinéraires

Littérature, textes, cultures

\title{
Anaphore résomptive conceptuelle et mémoire discursive : entre identité et altérité
}

\section{Mohamed Kara et Brigitte Wiederspiel}

\section{(2) OpenEdition}

\section{Journals}

Édition électronique

URL : http://journals.openedition.org/itineraires/134

DOI : 10.4000/itineraires.134

ISSN : 2427-920X

Éditeur

Pléiade

\section{Édition imprimée}

Date de publication : 1 juillet 2011

Pagination : 79-93

ISBN : 978-2-296-54673-8

ISSN : 2100-1340

\section{Référence électronique}

Mohamed Kara et Brigitte Wiederspiel, « Anaphore résomptive conceptuelle et mémoire discursive : entre identité et altérité », Itinéraires [En ligne], 2011-2 | 2011, mis en ligne le 10 janvier 2014, consulté le 20 avril 2019. URL : http://journals.openedition.org/itineraires/134; DOI : 10.4000/itineraires.134

\section{(C) $(\oplus \Theta$}

Itinéraires est mis à disposition selon les termes de la licence Creative Commons Attribution - Pas d'Utilisation Commerciale - Pas de Modification 4.0 International. 


\title{
Anaphore résomptive conceptuelle et mémoire discursive : entre identité et altérité
}

\begin{abstract}
In this paper, we propose to explore the relations between conceptual anaphora (ARC) and memory in a corpus of controversial discourse such as essays or lampoons. We try to show that these kinds of particular anaphora hold the same (idem) by the phenomenon of resumption but also a discursive otherness (ipse), in reference to Paul Ricœur's terminology. The anaphora conceptualization of the discursive situation will show us three strategies : definition, categorization and one-sided interpretation.
\end{abstract}

Keywords : anaphora, identity, conceptualization, memory and strategy Mots clés : anaphore, identité, conceptualisation, mémoire et stratégie

\section{Problématisation et méthodologie}

Que recouvre précisément le concept de «mémoire discursive »? Serait-il pertinent de lui associer le concept de « mémoire en discours ${ }^{1}$ » et dans quelle mesure les anaphores résomptives conceptuelles (définies infra) contribuent-elles à leur matérialisation? Cette double interrogation - qui est au principe de notre propos - nous conduira à examiner la manière dont un texte polémique ou d'orientation fortement argumentative se trame en associant de l'identité et de l'altérité cognitivo-discursive. Le choix des anaphores résomptives conceptuelles (dorénavant ARC) en tant que marqueur d'identité et d'altérité ne doit rien au hasard. Ces formes scandent le texte - les essais pour ce qui nous concerne - et intègre du « même » par le fait du phénomène de reprise engagé mais aussi de l'« altérité discursive » générée par l'activité de conceptualisation.

Quelle que soit la conception que l'on adopte du fait anaphorique - substitutive, antécédentiste ou encore mentaliste - conceptions maintes

1. Concept que nous empruntons à Marie-Anne Paveau (2006). 


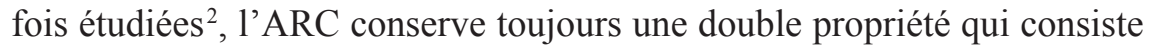
d'une part à rappeler des données posées ou présupposées et, d'autre part, à mettre à la disposition du lecteur un cadre définitionnel, catégorisant et/ ou interprétatif susceptible de varier selon le degré d'axiologisation de la reprise que l'on nommera "mémo-conceptuelle ». Un tel projet requiert un travail de définition de la mémoire discursive et de l'activité conceptuelle qui alimentent la problématique conjointe de l'identité et de l'altérité; définition subséquente aussi des ARC pour mieux en décrire les formes.

Sur un plan méthodologique, nous avons choisi de fonder nos investigations sur l'examen de quatre ouvrages relevant tous de scripteurs experts puisqu'il s'agit d'essayistes dont l'argumentativité se prête très bien à la controverse et donc à l'instauration de cadres discursifs marqués par le parti pris, en un sens non péjoratif du terme. Notre corpus est formé des quatre ouvrages suivants :

- Le Sanglot de l'homme blanc de Pascal Bruckner (1983)

- I.F. suite et fin de Régis Debray (2000)

- Les Communications humaines : pour en finir avec « la religion » de Régis Debray (2005)

- Le Monde d'après : une crise sans précédent de Matthieu Pigasse et Gilles Finchelstein (2009)

Pour chacun de ces ouvrages, nous nous sommes astreints à extraire automatiquement toutes les ARC, à souligner leur emplacement dans la topographie des textes pour mieux étudier leurs soubassements mémo-conceptuels.

\section{Anaphore et conceptualisation}

L'interprétation du lien anaphorique a successivement ouvert la voie à deux conceptions : une version traditionnelle où le texte est pris comme élément central de définition, puis une version mentaliste fondée sur des facteurs cognitifs donnant à la mémoire un rôle prépondérant.

Pour la première, la version « textuelle 3 » de l'anaphore qualifiée d' « antécédentiste » par Denis Apothéloz (cf. supra), l'identification du référent de l'expression anaphorique passe par la localisation d'un antécédent dans l'espace textuel : est anaphorique, tout item référentiel dont l'interprétation nécessite un appariement avec un élément linguistique du cotexte gauche. Cette définition repose sur une double restriction : la source linguistique doit non seulement précéder l'occurrence anaphorique mais aussi être essentielle à son interprétation.

2. Cf. la synthèse de Denis Apothéloz (1995: 307 sqq.).

3. Cf. Maillard (1974), Halliday et Hasan (1976), Fraser et Joly (1980), et Milner (1982). 
Pour la seconde conception, la version mentaliste ou « mémorielle ${ }^{4} »$, l'expression anaphorique n'est plus restreinte à la reconnaissance d'un élément textuel antécédent, mais s'étend au principe de cohérence, comme l'indication d'une continuité référentielle et cognitive du référent déjà manifeste dans la mémoire immédiate des locuteurs :

An expression occurs anaphorically if and only if it is interpreted with respect to an element in the preceding Contextuel Model, CMi [...] the listener keeps his attention directed to whatever it has been directed immediatly before the interpretation of the utterance in which the expression at issue occurs. (Bosh 1983: 64 et 89)

Une expression est anaphorique si et seulement si elle s'interprète relativement à un élément du Modèle Contextuel précédent [...] l'auditeur doit maintenir son attention sur l'élément mis en focus immédiatement avant l'interprétation de l'énonciation dans laquelle l'expression anaphorique en question apparait. (Nous traduisons)

Ce « Modèle Contextuel » est issu de l'interaction du traitement des enchaînements discursifs et des connaissances partagées. D'une certaine manière il s'apparente à la "mémoire discursive ${ }^{5}$ " telle qu'elle est évoquée par Alain Berredonner (qu'il convient de distinguer du concept de «mémoire discursive des textes » de Jean-Jacques Courtine, décrivant le rapport d'un texte à un autre, c'est-à-dire « l'existence historique de l'énoncé au sein de pratiques discursives réglées par des appareils idéologiques », 1981 : 53) et à la notion de " savoir partagé » de Marie-José Reichler-Béguelin en tant qu'ensemble de connaissances valides pour les interlocuteurs à l'instant $\mathrm{T}_{0}$.

L'avantage de la conception mémorielle est double pour le traitement des ARC. Tout d'abord, le référent visé par l'expression est une représentation mentale, un objet de mémoire et non plus un artefact extralinguistique. Ensuite, cette représentation mentale résulte d'une interaction entre des objets de mémoire immédiate, des recouvrements présuppositionnels et des inférences.

Sans en être l'analogue, notre définition de la mémoire discursive emprunte certains traits à celle de Reichler-Béguelin mais ne s'y réduit pas comme on le notera infra:

Nous appellerons mémoire discursive (= M) l'ensemble évolutif des connaissances officiellement partagées par les interlocuteurs. M est alimentée à la fois par les énonciations, par les percepts associés à la

4. Cf. Ehlich (1982), Bosch (1983) et Cornish (1990 et 2006).

5. Kleiber (1994:25) précise que la notion de « mémoire immédiate», est appelée diversement selon les auteurs, « univers de discours » (Lyons), « mémoire discursive » (Berrondonner et Reichler-Béguelin), « modèle contextuel » (Bosh), « modèle du discours » (Cornish) ou encore « focus » (Garrod et Sanford). 
situation d'énonciation, ainsi que par les inférences qui en découlent; y sont probablement reversées, au cas par cas, certaines connaissances à caractère culturel et encyclopédique, appartenant à un fonds scolaire estimé commun ${ }^{6}$.

Cette définition comporte une orientation communicationnelle en ce sens qu'elle souligne le caractère interactif et surtout évolutif des connaissances partagées. C'est dans cette dimension d'évolutivité que prennent corps les ARC qui, sous couvert de rappeler des informations antérieures constitutives de la mémoire discursive, introduisent de l'altérité via la conceptualisation.

« Conceptualiser », relève en effet d'une opération de reformulation qui consiste à instituer une relation présumée d'équivalence en gommant les spécificités des propositions inductrices pour ne retenir qu'un principe fédérateur emblématique des cas examinés; principe qui s'incarnera dans le nom correspondant. Le choix lexical impliqué est ici tout à fait déterminant puisque l'expression reformulante compactifiée se dote d'une vocation interprétative, propose un cadre interprétatif, ainsi que nous le verrons dans les exemples de notre corpus. Le terme retenu, un nom seul ou expansé, accomplit simultanément le résumé de la séquence première et la reformulation par un travail de généralisation duquel peut résulter un travail de définition proprement dit (ARC définitionnelle - paradigme restreint) ou une saisie axiologique - paradigme ouvert (ARC axiologique). Les ARC axiologiques, en ce sens, ne sont pas soumises exactement aux mêmes contraintes. Elles se distinguent notamment par le fait que le lien entre les propositions inductrices (cotexte gauche) et le reformulant est plus de nature inférentielle que strictement définitionnelle. La saisie nominale conceptuelle n'exploite que partiellement le thésaurus de toute la séquence princeps, en ne retenant qu'un nombre limité de traits inducteurs pour les convertir en une reformulation supposée plus englobante, plus aboutie que les premières formulations qui prennent dès lors le statut d'indices convergeant en faisceau et se résolvant ainsi dans l'anaphore résomptive conceptuelle qu'il nous appartiendra de définir et d'exemplifier plus rigoureusement. Notre définition rejoint d'une certaine manière celle de Guy Achard-Bayle qui considère que le conceptuel et la conceptualisation engagent une « matière qui, en dernier lieu, est configurée par l'esprit, donc déformée, manipulée, mais qui néanmoins tente de figurer (autrement dit : représenter) la portion du réel dont elle surgit, vient, nait ${ }^{7}{ }^{\prime}$.

Nous avons, pour notre part, proposé dans une contribution antérieure la définition suivante de la conceptualisation qui nous paraît conserver quelque pertinence :

Une séquence première, le plus souvent pluri-prédicative, de nature parfois descriptive, constituée de propositions inductrices à partir desquelles sera réalisée, dans une seconde séquence, la saisie conceptuelle sous la forme, pour le cas général, d'un syntagme nominal démonstratif et pour des cas

6. Reichler-Béguelin (1998: 97, note 6).

7. Cf. Achard-Bayle (2008: 61). 
plus particuliers, d'un syntagme nominal défini qui va mettre à disposition un cadre définitionnel et/ou interprétatif. (Kara et Wiederspiel 2007 : 110)

L'étude de quelques occurrences devrait nous permettre d'instruire le débat sur les propriétés mémorielles de la structure envisagée.

(1) Pourquoi le « parti intellectuel » ferait-il exception à l'implacable loi d'amochage qui tire le contraire du même? Ou doit-on supposer que nos dialecticiens hexagonaux sont immunisés contre la dialectique, et que le pendule de l'élection s'est arrêté sur les bords de Seine?

Pour dédramatiser un dépôt de bilan et panser notre amour-propre, il suffit de se remémorer les bénéfices initiaux et de prendre la courbe des opérations en son entier. La légende des origines se remâche et se ressasse, et c'est un peu nauséeux, à la fin; mais pour lassé qu'on soit de ces complaisances et de ces berceuses, nul n'a intérêt à jeter le berceau au feu s'il veut continuer à grandir. Il importe, au contraire, de rapporter l'état ultime d'une figure à son état princeps pour déceler ce qui les unit et les distingue. D'embrasser d'un même trait l'élan, l'inflexion et la chute; de reconnaître la continuité depuis le point de lancement sans déguiser la déconvenue du point d'arrivée. (Debray $2000: 12 / 13$ )

Dans cette occurrence, le syntagme nominal démonstratif joue un rôle interstitiel évident en matière de mémoire discursive. Les deux groupes coordonnés - ces complaisances, ces berceuses - font écho aux deux cotextes. Ils conceptualisent les groupes verbaux à gauche (dédramatiser, un dépôt de bilan, panser notre amour propre, se remémorer les bénéfices initiaux mais aussi se remâche et se ressasse) en les désignant comme des complaisances et des berceuses. Les mêmes phénomènes d'écho sont discernables à droite avec la constitution d'un plan isotope dont l'embrayeur est principalement le lexème berceuses. Les expressions suivantes assurent la continuité lexématique : jeter le berceau au feu, continuer à grandir ainsi qu'une certaine identité mémorielle. Il en va autrement du groupe nominal ces complaisances qui introduit une forme d'altérité discursive ou encore d'un surcroît conceptuel qui n'a pas d'ancrage direct dans les deux cotextes. C'est, entre autres choses, par le jeu de la coordination qu'il se trouve associé à ces berceuses sans en être l'analogue. On peut considérer que ces ARC, de par leur position à l'initiale de l'ouvrage préfigurent tout le jeu entre mémoire discursive - que l'on pourrait nommer identité thématique et problématique et altérité discursive par l'adjonction de points de vue non pas divergents mais différents, relevant en somme d'une sorte d'extranéité conceptuelle. C'est probablement en ce sens qu'il y a lieu de penser que cette anaphore n'est pas simplement résomptive. L'empan d'altérité discursive pourrait ainsi être un marqueur ou un constituant type de l'anaphore résomptive conceptuelle. Il semblerait, en première analyse, que les ARC constituent un point d'impact textuel qui assure les phénomènes de renvoi aux deux contextes mais aussi qui contribuent au renouvellement partiel du thématique et du problématique, notamment en lui adjoignant une dimension interprétative. 


\section{Coréférence et ipséité}

En référence à la terminologie de Paul Ricœur relative à l'« identité personnelle » et à l' " identité narrative »-pour peu bien sûr que l'on accepte la transposition à notre objet d'investigation -, les ARC se situeraient entre « mêmeté » et « ipséité ». Paul Ricœur considère à cet égard la mêmeté comme synonyme de l'identité-idem qu'il oppose à l'identité$i p s e^{8}$ qui, écrit-il, " n'implique aucune assertion concernant un prétendu noyau non changeant de la personnalité » (1990:13). Elles sont pourvues de certaines propriétés reformulantes sans être purement tautologiques : elles impliquent, outre du même, de la différence porteuse d'altérité discursive. Dans les ARC fortement axiologisées, nous pourrions presque parler d' « extranéité » dans la mesure où la saisie interprétative peut paraître, à certains lecteurs, abusive parce que non inférable du cotexte précédent et sujette à un coup de force interprétatif.

(2) (Zola 1898 : « Tout semble être contre moi, les deux Chambres, le pouvoir civil, le pouvoir militaire, les journaux à grand tirage, l'opinion publique qu'ils ont empoisonnée. Et je n'ai pour moi que l'idée... ») Comment? Par une course désespérée à la sanction, une lutte au couteau pour le bas $d u$ pavé, qui conduira par exemple à refiler dare-dare au petit camarade le sanbenito de la « bien-pensance » ou de la « pensée unique » qu'il vous a aimablement fourgué la veille, pour retrouver au plus vite sa solitude publique de mal pensant (de plus en plus difficile, par les temps qui courent). Boxe subversive, où le plus acculé gagne (le pendant du « judo moral »), plus incompris que moi tu meurs. D'où ces championnats de révolte où chacun, pour rester perdant, ne peut compter que sur son jeu de jambes. Pas grand sens de l'orientation, on en donne. Unique en son genre, copie conforme. Défenseur de Salman Rushdie et lanceur de fatwa. (Debray 2000 : 131-132)

Cette occurrence est emblématique du travail de conceptualisation dans la mesure où la reprise n'est que très peu prédictible, si l'on s'en tient toutefois aux prédications du cotexte gauche. Seul le paradoxe institué par les prédications suivantes « où le plus acculé gagne », ou encore «plus incompris que moi tu meurs » apprête le travail de conceptualisation qui va s'incarner dans l'ARC ainsi libellée : « D'où ces championnats de révolte » qui matérialise un concours de victimisation. Tout comme le réalise l'occurrence présentée ci-dessous :

(3) Juché sur des monceaux de cadavres propres à épouvanter quiconque, l'I.T. convoque aussitôt le « siècle d'Auschwitz et de la Kolyma » pour donner à son reportage l'ampleur attendue. Le ton apocalyptique, brutal, conquérant de la mise en demeure n'est pas seulement destiné à relancer et tenir en haleine un public distrait, assoupi ou lâche; il lui fait aussi sentir son indignité, et que le menu fretin n'est décidément pas à la hauteur. N'avonsnous pas tous besoin de croquemitaines pour éviter l'avachissement et nous

8. En référence à l'ipse latin. 
ressaisir enfin? L'inconvénient de ce procédé d'intimidation, d'intimation par l'hénaurme, c'est qu'il tourne souvent, chez le maniaque de l'agit-prop, à la drogue. Notre enfiévré, automate survolté, perd, à la fin, en force de persuasion ce qu'il a gagné en surface d'exposition. (Debray $2000: 68$ )

Le nom-tête, l'intimidation, ne s'impose pas sur la base d'un appariement avec un relevé sémique en cotexte gauche. Il procède d'un travail de recatégorisation, de remaniement conceptuel sur la base de traits co-occurrents (Le ton apocalyptique, brutal; la mise en demeure), mais aussi sur l'expression de l'indignité. Sur la base des exégèses précédentes, nous pourrions risquer la modélisation suivante du fonctionnement des ARC qui souligne l'oscillation entre mêmeté et ipséité discursive, d'altérité, voire d'extranéité conceptuelle.

\begin{tabular}{|c|c|c|c|c|}
\hline \multirow{5}{*}{$\begin{array}{l}\text { Formulation(s) } \\
\text { princeps. } \\
\text { (Dénomination } \\
\text { associée à des } \\
\text { prédications) }\end{array}$} & Reformulati & $\mathrm{n}$ interstitielle & ceptualisation & \multirow{5}{*}{$\begin{array}{l}\text { Formulation } \\
\text { remaniée, } \\
\text { incarnée dans } \\
\text { l'ARC } \\
\text { (Dénomina- } \\
\text { tion intersti- } \\
\text { tielle entre le } \\
\text { même et le } \\
\text { différent) }\end{array}$} \\
\hline & $\begin{array}{l}\text { Inscription / } \\
\text { attestation du posé / } \\
\text { présupposé }\end{array}$ & $\begin{array}{l}\text { Reprise avec } \\
\text { marques } \\
\text { résomptives }\end{array}$ & $\begin{array}{l}\text { Introduction dans un } \\
\text { champ conceptuel } \\
\text { nouveau }\end{array}$ & \\
\hline & \multicolumn{3}{|c|}{ Principe de mêmeté associé à de l'ipséité } & \\
\hline & \multicolumn{3}{|c|}{$\begin{array}{l}\text { Réitérer du même tout en introduisant, dans le travail de } \\
\text { conceptualisation, du nouveau qui relève de la manière de voir } \\
\text { du scripteur }\end{array}$} & \\
\hline & \multicolumn{3}{|c|}{$\begin{array}{l}\text { Principe d'altérité mémo-conceptuelle } \\
\text { (en particulier dans les reprises fortement axiologisées) }\end{array}$} & \\
\hline
\end{tabular}

Figure 1 : Tableau synoptique de la modélisation des ARC

\section{Particularités des ARC}

\section{Particularité nominale}

Dans les exemples précédents comme subséquents, les ARC se composent de noms syncatégorématiques à forte dépendance référentielle tels que le montrent ceux de nos extraits ci-avant « complaisances, berceuses ou championnats de révoltes » pareillement à ceux de notre corpus à venir « masochisme, sentiment ou vindicte ».

(4) Monde occidental, tu es condamné à mort

[...] Selon ce principe, tout ce qui élève, louange, célèbre l'Occident se voit soupçonné des pires noirceurs; en revanche, la modestie, l'humilité, le goût de l'autodestruction, ce qui peut incliner les Européens à s'éclipser, à rentrer dans le rang, est mis à l'honneur, salué comme hautement progressiste. La règle d'or de ce masochisme est simple : ce qui vient de nous est mauvais, ce qui vient d'autrui est parfait. (Bruckner 1983 : 47)

Le nom de l'ARC ce masochisme domine le cotexte par « abstraction catégorielle », laquelle établit une relation anaphorique dans la continuité 
d'un réseau de significations qu'elle subsume, en même temps qu'elle ajoute une variation, ce quelque chose de nouveau toujours déclenché par le déterminant démonstratif, comme l'ont indiqué les spécialistes de ce marqueur :

Les démonstratifs apportent toujours du nouveau : ils introduisent un nouveau référent ou un nouvel état du référent, ils changent le statut thématique du référent ou ils l'insèrent dans un nouvel univers. (de Mulder $1998: 30$ )

\section{Particularité textuelle}

Cette particularité est manifeste dans l'observation de nos exemples : l'ARC dépasse la limite structuraliste d'une séparation cotexte gauche versus cotexte droit et dynamise le rapport anaphore/cataphore. L'espace textuel est pris comme une «macro-entité linguistique » permettant des pointages en avant comme en arrière à l'intérieur même de sa structure (cf. l'exemple avec vindicte sans appel).

L'ARC subordonne toute une séquence textuelle à d'autres séquences par un jeu simultané de "subsomption » et de " recadrage ». Nous pourrions alors concevoir les ARC d'un texte polémique comme autant d'indications discursives qui jalonnent le raisonnement, en associant ces deux critères :

- SUBSOMPTION ou IDENTITÉ référentielle : le lien coréférentiel de l'ARC assure la remémoration des objets en discours;

- RECADRAGE Ou ALTÉRITÉ conceptuelle : l'élément nouveau nous paraît être avant tout de nature conceptuelle dans la mesure où il ne s'agit ni d'information inédite non inférable (cf. SNDP de Catherine Schnédecker) ni d'information supplémentaire ou détail additionnel d'une relation d'élaboration (cf. Kleiber et Vassiliadou 2009) ni même d'une modification d'état d'un référent, ni encore d'une variation de point de vue au sens où peut l'entendre Alain Rabatel (2003) lorsqu'il écrit :

On nommera PDV tout ce qui, dans la référenciation des objets (du discours) révèle, d'un point de vue cognitif, une source énonciative particulière et dénote, directement ou indirectement, ses jugements sur les référents.

L'ARC participe d'un recadrage de la situation, ou d'une réorientation du discours imposée par l'auteur, autrement dit d'un coup de force interprétatif sur ce qu'il faut retenir de la situation décrite en cotexte. Notre propos, en ce sens, rejoint celui d'Isabelle Delcambre :

Le cadre, précise-t-elle, est une façon de voir, il apparaît comme l'explicitation du sens donné par l'acteur à son action, il permet d'élucider le sens d'une situation pour les interactants, il contribue à la définition de la situation. Toute redéfinition d'une situation est un recadrage (2004:50). 


\section{Particularité mémorielle}

Il nous reste à préciser l'influence mémorielle dans l'interprétation des ARC de notre corpus d'essayistes. Un début d'explication, qui devra être vérifié in situ, est que leur interprétation passe nécessairement par une double exploitation : mémoire « des faits » et mémoire «immédiate des enchaînements textuels assurant la cohésion des discours ${ }^{9} »$.

Nous considérons ainsi que l'ARC procède par interaction entre :

- les connaissances partagées ou mémoire des faits, puisque l'ARC donne au lecteur un cadre interprétatif des positions énonciatives sur une situation politico-sociale donnée;

- et une mémoire immédiate des enchaînements textuels, pour la continuité anaphorique qu'elle conforte comme unité de discours fondamentalement dépendante ou « satellite » au sens de Francis Cornish (2006).

De ce fait, plutôt que de parler de mémoire discursive au sens restreint de Alain Berrendonner - qui résulte d'un savoir partagé nécessaire à l'interaction scripteur-lecteur - ou de mémoire discursive au sens large de JeanJacques Courtine (cité par Paveau 2006 : 95) « qui concerne l'existence historique de l'énoncé au sein de pratiques discursives réglées par des appareils idéologiques », nous préférons reconnaître, à la suite de MarieAnne Paveau (2006), le processus dynamique d'une mémoire « cognitivodiscursive » ou « mémoire en discours » en raison du travail de conceptualisation que les ARC réalisent.

\begin{tabular}{|c|c|c|c|}
\hline \multirow{2}{*}{ 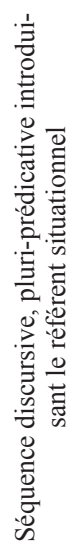 } & $\begin{array}{l}\quad \text { Remémoration } \\
\text { Indication d'une continuité réfé- } \\
\text { rentielle cognitive }\end{array}$ & $\begin{array}{l}\text { Recadrage cognitivo-discursif } \\
\text { Nouvelle conceptualisation d'une situation : soit } \\
\text { les } 3 \text { stratégies discursives les plus remarquables } \\
\text { dans nos corpus }\end{array}$ & \multirow[b]{2}{*}{$\begin{array}{l}\mathrm{A} \\
\mathrm{R} \\
\mathrm{C}\end{array}$} \\
\hline & $\begin{array}{l}\text { Articulation des enchaînements } \\
\text { textuels de la séquence; continuité } \\
\text { d'un plan isotope; } \\
\text { Saillance : le référent situationnel } \\
\text { devient manifeste dans la } \\
\text { mémoire immédiate du lecteur; } \\
\text { Démarcation : délimitation des } \\
\text { étapes d'une analyse ou d'une } \\
\text { pensée : élection d'une position } \\
\text { énonciative. }\end{array}$ & 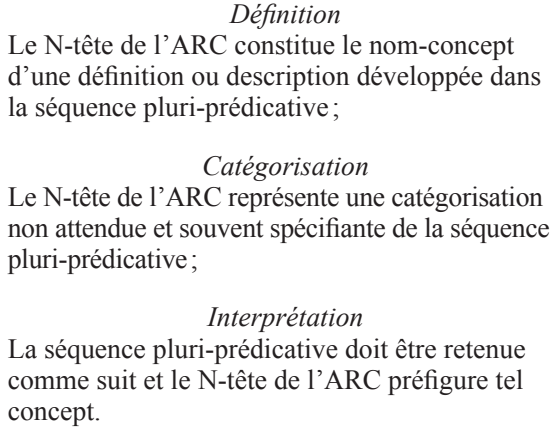 & \\
\hline & & $\begin{array}{l}\text { Gradation ou processus scalaire } \\
\text { du plus assertorique au plus axiologisé. } \\
\text { Réinterprétation unilatérale, } \\
\text { ou plus ou moins forcée, de la situation. }\end{array}$ & \\
\hline
\end{tabular}

Figure 2 : Tableau synoptique sur la mémoire discursive des ARC

9. Cf. Paveau (2006: 86 et 97-98). 


\section{Application sur typologies constrastives}

\section{Essais polémiques ou remémoration stratégique}

Nous commencerons par un ample passage de Pascal Bruckner, qui s'inscrit dans un sous-chapitre d'une dizaine de pages avec pour thématique la guerre du Vietnam. Quelques ARC jalonnent le discours et jouent un rôle éminemment démarcatif dans la progression du raisonnement. Elles imposent, nous semble-t-il, la stratégie idéologique de l'écrivain, c'està-dire son mode d'interprétation des faits : définitionnel, catégorisant ou interprétatif.

(5) Chap. 1 - La solidarité ou la légende noire contre l'histoire sainte Sous-chap. «L'Amérique a la rage »

\section{$1^{\text {re } A R C ~: ~ D e ́ f i n i t i o n ~}$}

Une certitude confuse, exprimée de manière plus ou moins nuancée selon les familles politiques [...], anime ceux qu'on allait appeler les tiersmondistes : celle de l'infamie de l'Occident, [...]. L'Occident est prédateur, [...]. C'est pourquoi un fossé divisait le royaume du salut total du royaume du mal total, entre lesquels il ne peut y avoir que l'adhésion ou la répudiation. C'est pourquoi encore, en soutenant les peuples que l'Europe avait, jusqu'il y a peu, tenus sous sa botte, on pouvait gommer ou du moins atténuer l'atroce épisode de la prépondérance. Ce sentiment d'une dette impossible à éponger, nul mieux que Sartre, $[\ldots]$ ne devait le susciter et le fonder en droit. (Bruckner 1983 : 31-32)

L'ARC Ce sentiment d'une dette impossible à éponger active la mémoire immédiate de la séquence mais également rappelle l'idée centrale développée dans l'essai, à savoir l'impact de notre passé colonialiste et impérialiste.

On reconnaît dans cette ARC une structure définitionnelle fondée sur une série d'antonymies discursives salut total vs mal total; adhésion vs répudiation dont l'un des termes appartient forcément à une position énonciative différente de celle de l'auteur. Nous pourrions proposer la glose suivante :

Certaines familles politiques, exacerbant dans leur discours l'infamie de l'Occident dans l'atroce épisode de sa prépondérance, à savoir le colonialisme et l'impérialisme, entretiennent l'idée que tout Occidental éprouve un sentiment de dette impossible à éponger.

\section{2e ARC : Catégorisation}

(6) D'après lui, les crimes que l'on commet en notre nom, il faut bien que nous en soyons personnellement complices puisqu'il reste en notre pouvoir de 
les arrêter : Cette culpabilité qui reposait en nous inerte, étrangère, il faut bien $[\ldots]$ que nous nous avilissions nous-mêmes pour pouvoir la supporter. (Ibid. : 32)

La $2^{\mathrm{e}} \mathrm{ARC}$ de cet extrait, sous-catégorise la première et guide le lecteur dans son interprétation. Nous continuerons donc par cette autre glose qui tient compte de l'ensemble du passage :

Ce sentiment de dette impossible à éponger, au lieu de générer, comme on serait en droit de le penser, des engagements ou des actions envers les peuples opprimés pour tenter de solder ce passif, s'est converti en culpabilité qui plus est inerte et étrangère, c'est-à-dire vaine et insensible.

\section{$3^{e}$ ARC : Réorientation du discours, recadrage cognitivo-discursif axiologisé et fonction interstitielle}

(7) Du coup, l'exécration intérieure que se vouait l'Européen cessait de tourner à l'autoflagellation et se déportait sur un tiers, symbole du crime absolu. Mais il fallait un tiers assez proche, qui réunisse en lui tous les traits que nous détestons chez nous. Le souvenir de l'effondrement colonial était encore vivace, un vieux peuple historique cessait d'exister comme prédominant et en concevait un immense complexe d'infériorité : l'Amérique offrait alors le spectacle hallucinant d'une grande puissance occidentale qui recommençait l'aventure impérialiste quand toutes les métropoles avaient dit pouce.

Rage ou crainte de la voir réussir là où il venait d'échouer, le Vieux Continent effectua une distribution manichéenne des rôles : cette vindicte sans appel fut alors vécue par chacun comme une impérieuse religion nationale. L'Américain était maudit en raison de la déviation minuscule qu'il représentait vis-à-vis de l'Européen, frère ennemi, presque semblable, différent de nous, mais à peine, et on lui en voulait pour cet écart. La haine s'adressait au parent, à l'intime dont on désavouait l'insupportable proximité. L'Amérique, fille dénaturée, concentrait tous les traits négatifs de ses patries d'origine : robotisation, machinisme, matérialisme, angoisse. Double de l'Europe peut-être, mais au sens où les parents les plus sains peuvent enfanter des monstres. (Ibid. : 39)

L'interprétation de l'ARC cette vindicte sans appel traverse toute la séquence et y joue prototypiquement une fonction interstitielle. D'une part, elle fait référence à la proposition précédente immédiate - le fait que le Vieux Continent effectua une distribution manichéenne des rôles. D'autre part, elle tisse des liens isotopiques en cotextes plus larges. Cette ARC rappelle les expressions nominales du cotexte gauche (culpabilité inerte, exécration intérieure, autoflagellation, complexe d'infériorité) pour mieux s'en dégager : la déculpabilisation de l'Europe emprunte un autre chemin que celui prédictible, comme l'indique P. Bruckner, elle va se déporter sur l'Amérique (rage, Américain maudit, frère ennemi, haine, fille dénaturée, traits négatifs, monstres) avec en arrière-plan le conflit vietnamien. 
La position énonciative que décrit la dénomination cette vindicte expansée de la qualification juridique sans appel n'appartient pas aux familles politiques évoquées; son champ lexical est sensiblement différent de celui de haine ou exécration. Nous y reconnaissons un écart de l'ordre d'une réorientation du discours (Fossard : 83) que nous commenterons comme suit :

En énonçant cette vindicte sans appel le locuteur-narrateur nous livre, sous couvert d'une marque d'objectivité, sa propre conception de la situation : la culpabilité de l'Occident liée à ses méfaits colonialistes et impérialistes s'est déportée ou déplacée, comme une vengeance, en haine justifiée envers les Américains - une vengeance déguisée en justice simplificatrice qui oppose sans nuance le bien et le mal.

La stratégie de l'ARC consiste à nous imposer ce recadrage du discours, comme un coup de force interprétatif : stratégie plutôt récurrente dans cet essai, qui serait classé comme " prose d'idées ", ou encore " littérature d'idées » par Marc Angenot, ce qui caractérise le style singulier des essayistes.

\section{Essais d'investigation ou persistance mémorielle}

De façon remarquablement différente, Matthieu Pigasse et Gilles Finchelstein nous proposent, dans un essai peu polémique, une analyse technique et pédagogique sur les données de la crise financière actuelle.

Il nous a semblé patent que cet essai d'investigation à visée objectivante présenterait des situations discursives et conceptuelles opposées dans la mesure où il détermine des stratégies liées davantage à l'entendement et à la connaissance plutôt qu'à la persuasion. Effectivement, ne renfermant pratiquement pas d'ARC, ce texte est au contraire saturé d'anaphores fidèles et de quelques anaphores résomptives :

(8) Chronologiquement, la crise s'est donc propagée par un effet domino. La chute des prix de l'immobilier a conduit de nombreux ménages emprunteurs à devenir insolvables. Cette insolvabilité s'est traduite par des pertes dans les banques. Ces pertes dans les banques ont conduit à une restriction du crédit. Ces restrictions de crédit se traduisent par une augmentation du coût de financement des entreprises et une diminution de la croissance. (Pigasse et Finchelstein $2009: 67$ )

Les anaphores de cet ouvrage présentent les particularités textuelles et cognitivo-référentielles suivantes :

- PROXIMITÉ : les éléments repris précèdent immédiatement l'expression démonstrative;

- IDENTITÉ FORMELLE : les anaphores fidèles sont parfois de véritables répétitions, avec maintien de l'expansion de la mention antérieure, pour pallier toute difficulté de remémoration ou toute rupture du lien référentiel;

- RÉSOMPTION non axiologisée : la dérivation est le cas le plus fréquent pour expliquer l'AR. 


\section{Conclusion}

En guise de conclusion provisoire, un autre aspect mérite d'être mis en évidence. Les ARC sont typiquement des poly-opérateurs au sens que leur donne Denis Apothéloz (1995 : 54), plus encore que les anaphores " ordinaires ", si l'on peut dire. Par poly-opérateur, il convient de comprendre que le rappel anaphorique, en pareil cas, n'est pas uniquement proposé pour communiquer « au destinataire l'identité du référent : le locuteur peut mettre à profit le rappel anaphorique pour accomplir en parallèle diverses opérations, qu'elles soient d'ordre pragmatique, planificatoire ou interactif $\gg$. Cela signifie concrètement qu'il s'agit de syntagmes pourvus de plusieurs propriétés textuelles et cognitivo-conceptuelles. Les dénombrer de façon exhaustive serait tâche fastidieuse mais l'on peut toutefois en proposer les principales facettes qui montrent toute la complexité de fonctionnement des ARC. On peut ainsi discerner :

- Une fonction d'étalement syntaxique, au sens où le propose Halliday et Hasan (1976) qui permet de déployer une information, un raisonnement, une conceptualisation.

- Une fonction d'activation/réactivation mémorielle, spécialement en charge de la mémoire discursive lorsqu'elle est menacée d'extinction, notamment par les effets de saillance cognitive.

- Une fonction interstitielle entre le cotexte gauche et le cotexte droit. Il semble avéré que les ARC servent aussi - peut-être surtout - à mettre en saillie une information nouvelle. C'est en quelque sorte une fonction d'ancrage rhématique.

- Une fonction de modalisation qui a l'avantage de régler le degré de prise en charge de l'énoncé par le rédacteur; bref, d'ajuster le propos entre le pôle assertorique et le pôle modalisé, autrement dit entre l'information tendant à la neutralité et celle plus orientée sur un versant plus engagé, voire axiologique.

- Une fonction de focalisation, d'emphase, d'additif référentiel, etc.

- Une fonction de réinstanciation orientée d'un objet de discours (ODD).

- Une fonction de guidage à l'interprétation qui opère via l'institution d'une mémoire discursive commune.

Mohamed Kara et Brigitte Wiederspiel Université Paul Verlaine - Metz, CELTED 


\section{Références}

Achard-Bayle, G., 2008, Les réalités conceptuelles. Identité et/en fiction, Metz, Université Paul Verlaine, coll. « Recherches Textuelles », no 8.

Angenot, M., 1982, La parole pamphlétaire, Paris Payot.

Bosch, P., 1983, Agreement and Anaphora. A Study of the Role of Pronouns in Syntax and Discourse, London, Academic Press.

Cornish, Fr., 1990, « Anaphore pragmatique, référence, et modèle du discours », dans G. Kleiber et J.-E. Tyvaert (dir.), L'Anaphore et ses domaines, Université de Metz, coll. « Recherches linguistiques », n 14, p. 81-96.

—, 2006, «Relations de cohérence en discours : critères de reconnaissance, caractérisation et articulation cohésion-cohérence », CORELA Numéros thématiques, Organisation des textes et cohérence des discours, http://edel.univ-poitiers.fr/corela/document.php $? \mathrm{id}=1280 \&$ format=print (consulté le 10 juin 2010).

Courtine, J.-J., 1981, «Quelques problèmes théoriques et méthodologiques en analyse du discours, à propos du discours communiste adressé aux chrétiens », Langages, $n^{\circ} 62$, p. 9-128.

Delcambre, I., 2004, « Cadre et cadrage discursifs : problèmes d'écriture théorique », Pratiques, ${ }^{\circ}$ 121-122, p. 45-57.

De Mulder, W., 1998, « Du sens des démonstratifs à la construction d'univers », Langue Française, n 120 , p. 5-21.

Ehlich, K., 1982, « Anaphora and Deixis : Same, Similar or Different? », dans R. Jarvella et W. Klein (dir.), Speech, Place and Action, Chichester, John Wiley and Sons, p. 315-338.

Fossard, Marion, 2006, « Aspects psycholinguistiques du traitement des démonstratifs : résultats croisés en français et en anglais », Langue Française, $\mathrm{n}^{\circ} 152$, p. 82-95.

Fraser, Th. et Joly, A., 1980, « Le système de la deixis, endophore et cohésion discursive en anglais ", Modèles linguistiques, $\mathrm{n}^{\circ}$ II-2, p. 22-51.

Halliday, M. A. K. et Hasan, R., 1976, Cohesion in English, London-New York, Longman.

Kara, M. et Wiederspiel, Br., 2007, « Anaphores résomptives et reformulations ", dans M. Kara (dir.), Usages et analyses des reformulations, Metz, Université de Metz, coll. « Recherches linguistiques », n² 29, p. 97-121.

—, 2010, «Choix lexicaux dans les reprises anaphoriques conceptuelles », dans Directions actuelles en linguistique du texte. Le Texte : modèles, méthodes, perspectives, Cluj-Napoca, Casa Cartii de Stiinta, p. 200-212.

Kleiber, G., 1994, Anaphores et pronoms, Louvain-la-Neuve, Duculot. 
Kleiber, G. et Vassiliadou, H., 2009, « Sur la relation d'Élaboration : des approches intuitives aux approches formelles », Journal of French Language Studies, ${ }^{\circ}$ 19, Special Issue 2, p. 183-205.

Maillard, M., 1974, "Essai de typologie des substituts diaphoriques », Langue française, $\mathrm{n}^{\circ} 21$, p. 55-71.

Milner, J.-Cl., 1982, « Coréférences et anaphores », dans Ordres et raisons de langue, Paris, Seuil, p. 31-42.

Paveau, M.-A., 2006, Les prédiscours. Sens, mémoire, cognition, Paris, Presses de la Sorbonne nouvelle.

—, 2007, « Discours et cognition. Les prédiscours entre cadres internes et environnement extérieur », Corela - Numéros thématiques, Contextes, discours, cognitions, http://edel.univ-poitiers.fr/corela/document. php ?id=1672 (consulté le 10 juin 2010).

Rabatel,A., 2003, « Pour une narratologie énonciative ou pour une approche énonciative de la narration ", Vox Poetica La narratologie aujourd'hui, http://www.vox-poetica.org/t/lna/rabatel.htm, (consulté le 10 juin 2010).

Ricoeur, P., 1990, Soi-même comme un autre, Seuil.

Reichler-Béguelin, M.-J., 1998, «L'usage des SN démonstratifs dans les Fables de La Fontaine », Langue Française, n 120, p. 95-109.

—, 1989, «Anaphores, connecteurs et processus inférentiels », dans C. Rubattel (dir.), Modèles du discours. Recherches actuelles en Suisse romande, Berne, Peter Lang, p. 303-336.

Schnedecker, C., 2006, "SN démonstratif "prédicatifs" : qu'est-ce qui limite leur apport informatif? », Langue Française, n 152, p. 39-55. 